\title{
ON COMPACT OPERATORS IN THE WEAK CLOSURE OF THE RANGE OF A DERIVATION
}

\author{
HONG W. KIM ${ }^{1}$
}

\begin{abstract}
It is shown that if $K$ is a compact operator which commutes with a bounded operator $A$ on a Hilbert space $H$ and if $K$ is contained in the weak closure of the range of the derivation induced by $A$, then $K$ is quasinilpotent.
\end{abstract}

Introduction and notations. Let $H$ be a fixed separable infinite dimensional Hilbert space over the complex field $C$, and let $\delta_{A}(X)=A X-X A$ be an inner derivation on the algebra $\mathscr{L}(H)$ of all bounded operators on $H$. $R\left(\delta_{A}\right)^{-}$will denote the norm closure of the range of $\delta_{A}$ in $\mathscr{L}(H)$, and $R\left(\delta_{A}\right)^{-w}$ will denote the closure of $R\left(\delta_{A}\right)$ in the weak operator topology. $\{A\}^{\prime}$ will denote the commutant of $A$.

Kleinecke (1957) and Shirokov (1956) independently proved I. Kaplansky's conjecture that $R\left(\delta_{A}\right) \cap\{A\}^{\prime}$ consists entirely of quasinilpotent operators.

In fact, using their result, it is rather simple to show that if $A X_{n}-X_{n} A$ converges to $B$ in $\{A\}^{\prime}$ in the norm topology for $\left\|X_{n}\right\| \leqq M,(M>0)$, then $B$ is again quasinilpotent, as Pearcy has noted in [6].

Recently, J. Anderson [1] proved that there exists an operator $A$ in $\mathscr{L}(H)$ such that $I \in R\left(\delta_{A}\right)^{-}$. Therefore, an operator $B$ in $R\left(\delta_{A}\right)^{-} \cap\{A\}^{\prime}$ is not necessarily quasinilpotent.

The purpose of this paper is to prove that if $\left\{X_{n}\right\}$ is a sequence of operators such that $\left(A X_{n}-X_{n} A\right) \rightarrow K$ in the weak operator topology where $K$ is a compact operator in $\{A\}^{\prime}$, then $K$ is quasinilpotent.

Lemma. Let $A \in \mathscr{L}(H)$ and let $\left\{X_{n}\right\}$ be a sequence of operators in $\mathscr{L}(H)$ such that the sequence $\left\{A X_{n}-X_{n} A\right\}$ converges weakly to an operator $C$ in

Presented to the Society, October 28, 1972 under the title On compact operators in the norm closure of the range of an inner derivation; received by the editors October 9, 1972 and, in revised form, December 20, 1972.

AMS (MOS) subject classifications (1970). Primary 47B47, 47B05.

Key words and phrases. Range of a derivation, eigenvalues, quasinilpotent operators, norm closure, weak operator topology, commutant of an operator, compact operator.

1 The author wishes to express his gratitude to Professor Carl Pearcy for many helpful suggestions concerning this paper during his stay at Bucknell Univerity as a Distinguished Visiting Professor. This research was supported, in part, by a Bucknell University Summer Research Grant in 1972.

(c) American Mathematical Society 1973 
$\{A\}^{\prime}$. Then, $(A-\lambda)^{j} X_{n}-X_{n}(A-\lambda)^{j} \rightarrow j(A-\lambda)^{j-1} C$ in the weak operator topology for every positive integer $j$ and every fixed scalar $\lambda$. (It is assumed that $(A-\lambda)^{j}=$ I if $j=0$.)

Proof by Induction. The case $j=1$ is obvious.

For $j=2$, multiply the commutator $A X_{n}-X_{n} A=(A-\lambda) X_{n}-X_{n}(A-\lambda)$ on the left by $A-\lambda$ and add it to the commutator multiplied on the right by $A-\lambda$. Then, $(A-\lambda)^{2} X_{n}-X_{n}(A-\lambda)^{2} \rightarrow 2(A-\lambda) C$ weakly. Suppose $(A-\lambda)^{k} X_{n}-X_{n}(A-\lambda)^{k} \rightarrow k(A-\lambda)^{k-1} C$ weakly for all $k \leqq j$ where $j \geqq 2$.

Then

$$
\begin{aligned}
(A-\lambda)\{( & \left.(A-\lambda)^{j} X_{n}-X_{n}(A-\lambda)^{j}\right\}+\left\{(A-\lambda)^{j} X_{n}-X_{n}(A-\lambda)^{j}\right\}(A-\lambda) \\
= & (A-\lambda)^{j+1} X_{n}-X_{n}(A-\lambda)^{j+1} \\
& +(A-\lambda)\left\{(A-\lambda)^{j-1} X_{n}-X_{n}(A-\lambda)^{j-1}\right\}(A-\lambda) \\
\rightarrow & 2 j(A-\lambda)^{j} C \text { weakly. }
\end{aligned}
$$

Since $(A-\lambda)^{j-1} X_{n}-X_{n}(A-\lambda)^{j-1} \rightarrow(j-1)(A-\lambda)^{j-2} C$ weakly by the induction assumption, $(A-\lambda)^{j+1} X_{n}-X_{n}(A-\lambda)^{j+1} \rightarrow(j+1)(A-\lambda)^{j} C$ weakly.

TheOREM 1. Let $A \in \mathscr{L}(H)$ and let $\left\{X_{n}\right\}$ be a sequence of operators in $\mathscr{L}(H)$ such that the sequence $\left\{A X_{n}-X_{n} A\right\}$ converges weakly to an operator $C$ of finite rank in $\{A\}^{\prime}$. Then $C$ is nilpotent.

Proof. Let the range of $C$ be $R(C)$ and let $\operatorname{dim} R(C)=k \geqq 1$. If $R\left(C^{i+1}\right) \neq R\left(C^{i}\right)$ for $1 \leqq i \leqq k$, then clearly $C$ is nilpotent. Hence, we assume the existence of the smallest positive integer $q$ such that $R\left(C^{q}\right)=R\left(C^{q+1}\right)$. Then, $R\left(C^{q}\right)=R\left(C^{r}\right) \neq\{0\}$ for all $r \geqq q$ and $C$ restricted to $R\left(C^{q}\right)$ is nonsingular. Also, $A$ may be assumed to be nonsingular by replacing $A$ by $A-\lambda I$ if necessary. For notational convenience, we let $R\left(C^{q}\right)=W$. Since $C \in\{A\}^{\prime}, W$ is invariant under $A$ and $A(W)=W$.

Let $\lambda_{1}, \lambda_{2}, \cdots, \lambda_{p}$ be the distinct eigenvalues of $A \mid W$ (i.e., $A$ restricted to $W)$ with respective algebraic multiplicities $m_{1}, m_{2}, \cdots, m_{p}$. Then, by Jordan's theorem $W$ is a direct sum (not necessarily orthogonal) of $p$ subspaces $M_{1}, M_{2}, \cdots, M_{p}$ with respective dimensions $m_{1}, m_{2}, \cdots, m_{p}$ such that $A M_{i} \subseteq M_{i}$ and $A-\lambda_{i}$ is nilpotent on $M_{i}$ with index $j_{i} \leqq m_{i}$ for $i=1,2, \cdots, p$.

Since the index of $A-\lambda_{1}$ on $M_{1}$ is $j_{1} \geqq 1$, there exists a vector $y \in M_{1}$ such that $\left(A-\lambda_{1}\right)^{j_{1}-1} y \neq 0$ and $\left(A-\lambda_{1}\right)^{j_{1}} y=0$. The fact that $A X_{n}-X_{n} A \rightarrow C$ weakly implies that $C^{q} A X_{n}-C^{q} X_{n} A=A\left(C^{q} X_{n}\right)-\left(C^{q} X_{n}\right) A \rightarrow C^{q+1}$ weakly. By the previous lemma,

$$
\left(A-\lambda_{1}\right)^{j_{1}}\left(C^{q} X_{n}\right)-\left(C^{q} X_{n}\right)\left(A-\lambda_{1}\right)^{j_{1} \rightarrow j_{1}}\left(A-\lambda_{1}\right)^{j_{1}-1} C^{q+1}
$$

weakly. 
Since $\left(C^{q} X_{n}\right) y \in W$, it is obvious that

$$
\left(A-\lambda_{1}\right)^{j_{1}}\left(C^{q} X_{n}\right) y \in M_{2} \oplus M_{3} \oplus \cdots \oplus M_{p} .
$$

Now $j_{1} C^{q+1}\left(A-\lambda_{1}\right)^{j_{1}-1} y$ is a nonzero vector in $M_{1}$, because of the way $y$ was chosen and the nonsingularity of $C^{q+1} \mid W$.

Since $M_{1}, M_{2}, \cdots, M_{p}$ are fixed and $\left(A-\lambda_{1}\right)^{j_{1}} y=0$,

$$
\inf _{n}\left\|\left(A-\lambda_{1}\right)^{j_{1}}\left(C^{q} X_{n}\right) y-j_{1} C^{q+1}\left(A-\lambda_{1}\right)^{j_{1}-1} y\right\|>0 .
$$

On the other hand, since $W$ is finite dimensional and is invariant under $\left(A-\lambda_{1}\right)^{j_{1}}\left(C^{q} X_{n}\right),\left(C^{q} X_{n}\right)\left(A-\lambda_{1}\right)^{j_{1}}$, and $\left(A-\lambda_{1}\right)^{j_{1}-1} C^{q+1}$ for all $n=1,2, \cdots$, $\left(A-\lambda_{1}\right)^{j_{1}}\left(C^{q} X_{n}\right)-\left(C^{q} X_{n}\right)\left(A-\lambda_{1}\right)^{j_{1}} \mid W$ converges strongly to

$$
j_{1}\left(A-\lambda_{1}\right)^{j_{1}-1} C^{q+1} \mid W .
$$

Therefore,

$$
\lim _{n}\left\|\left(A-\lambda_{1}\right)^{j_{1}}\left(C^{q} X_{n}\right) y-j_{1} C^{q+1}\left(A-\lambda_{1}\right)^{j_{1}-1} y\right\|=0 .
$$

This is an obvious contradiction. Hence, all operators of finite rank in $R\left(\delta_{A}\right)^{-w} \cap\{A\}^{\prime}$ are nilpotent.

This result allows us to obtain the following stronger theorem.

THEOREM 2. If $A \in \mathscr{L}(H)$ and $\left\{X_{n}\right\}$ is a sequence of operators in $\mathscr{L}(H)$ such that $\left(A X_{n}-X_{n} A\right)$ converges weakly to a compact operator $K$ in $\{A\}^{\prime}$, then $K$ is quasinilpotent.

Proof. Suppose $K$ is not quasinilpotent. Then, there exists an isolated nonzero eigenvalue $\lambda_{0}$ in the spectrum $\sigma(K)$ of $K$. By a theorem of Riesz [7, p. 183], the root space $M\left(\lambda_{0}\right)$ corresponding to $\lambda_{0}$ of $K$ (i.e., the subspace $\left\{f \in H:\left(K-\lambda_{0}\right)^{n} f=0\right.$ for some positive integer $\left.\left.n\right\}\right)$ is finite dimensional, is invariant under $K$, and has the property that $K \mid M\left(\lambda_{0}\right)$ has singleton spectrum $\left\{\lambda_{0}\right\}$. Since $\sigma(K)$ is denumerable with 0 the only possible point of accumulation, it is possible to choose two disjoint simply connected open sets $\mathscr{U}_{1}$ and $\mathscr{U}_{2}$ such that $\lambda_{0} \in \mathscr{U}_{1}$ and $\sigma(K)-\left\{\lambda_{0}\right\} \subset \mathscr{U}_{2}$. Let $\gamma_{1}$ be a simple closed rectifiable curve lying in $\mathscr{U}_{1}$ containing $\lambda_{0}$ in its interior, and let $\gamma_{2}$ be a simple closed rectifiable curve lying in $\mathscr{U}_{2}$ containing $\sigma(K)-\left\{\lambda_{0}\right\}$ in its interior.

By a theorem of Riesz [7, p. 421],

$$
E=\frac{1}{2 \pi i} \int_{\gamma_{1}}(z-K)^{-1} d z
$$


is an idempotent which commutes with $K$, and the range of $E$ is $M\left(\lambda_{0}\right)$. Let $f(z)$ be the analytic function defined on $\mathscr{U}_{1} \cup \mathscr{U}_{2}$ which is identically 1 on $\mathscr{U}_{1}$ and identically 0 on $\mathscr{U}_{2}$. It follows from the definition of $f(K)$ [7, p. 431] and (1) that $f(K)=E$. Since the complement of $\mathscr{U}_{1} \cup \mathscr{U}_{2}$ is connected in the extended complex plane, it follows from Rung's theorem [4, p. 317] that there exists a sequence of polynomials $P_{n}(z)$ which converges uniformly to $f(z)$ on $\gamma_{1} \cup \gamma_{2}$.

Thus,

$$
\begin{aligned}
P_{n}(K)-E= & P_{n}(K)-f(K) \\
= & \frac{1}{2 \pi i} \int_{\gamma_{1}}\left(P_{n}(z)-f(z)\right)(z-K)^{-1} d z \\
& +\frac{1}{2 \pi i} \int_{\gamma_{2}} P_{n}(z)(z-K)^{-1} d z .
\end{aligned}
$$

Since $\left\|(z-K)^{-1}\right\|$ is uniformly bounded on $\gamma_{1} \cup \gamma_{2}$, it follows that $\left\|P_{n}(K)-E\right\| \rightarrow 0$. Now observe that the sequence $K\left(A X_{n}-X_{n} A\right)=A\left(K X_{n}\right)$ $-\left(K X_{n}\right) A$ converges weakly to $K^{2}$. A similar argument shows that if $P$ is any polynomial, then $P(K)$ belongs to $R\left(\delta_{A}\right)^{-w} \cap\{A\}^{\prime}$. In particular, if $P_{n}(z)$ is the sequence determined above, then $P_{n}(K) \in R\left(\delta_{A}\right)^{-w} \cap\{A\}^{\prime}$. Therefore, $f(K)=E \in R\left(\delta_{A}\right)^{-w} \cap\{A\}^{\prime}$. Since $E$ is an operator of finite rank and $1 \in \sigma(E)$, this contradicts Theorem 1. Therefore all compact operators $K$ in $R\left(\delta_{A}\right)^{-w} \cap\{A\}^{\prime}$ are quasinilpotent.

Examination of the proof of Theorem 2 shows that the hypothesis that $K$ is compact was used only to conclude that the idempotent $E$ defined by (1) is an operator of finite rank. Thus, we have actually proved the following stronger result.

TheOREM 3. Let $A \in \mathscr{L}(H)$ and let $T$ be any operator in $R\left(\delta_{A}\right)^{-w} \cap\{A\}^{\prime}$. If $\lambda_{0}$ is any isolated point in $\sigma(T)$, and $\gamma$ is a simple closed rectifiable curve in the resolvent set of $T$ containing, in its interior, no other points of $\sigma(T)$ except $\lambda_{0}$, then the idempotent $E=(2 \pi i)^{-1} \int_{\gamma}(z-T)^{-1} d z$ must have infinite dimensional range.

Some remarks. In [10] J. P. Williams proves that if $A$ and $B$ belong to $\mathscr{L}(H)$ and are not scalar multiples of the identity, then $R\left(\delta_{A}\right) \cap R\left(\delta_{B}\right) \neq 0$. Therefore, $R\left(\delta_{A}\right)$ is in general large and contains a subset of compact operators. But, in [9], Stampfli proved that no $R\left(\delta_{A}\right)$ is large enough to contain the ideal $F$ of all finite rank operators. The problem of classifying all noncompact operators in $R\left(\delta_{A}\right)^{-10} \cap\{A\}^{\prime}$ for a given $A$ in $\mathscr{L}(H)$ is still an open problem. 


\section{BIBLIOGRAPHY}

1. J. Anderson, Derivation ranges and the identity (to appear).

2. I. C. Gohberg and M. G. Krein, Introduction to the theory of linear nonselfadjoint operators in Hilbert space, "Nauka", Moscow, 1965; English transl., Transl. Math. Monographs, vol. 18, Amer. Math. Soc., Providence, R.I., 1969. MR 36 \#3137; 39 \#7447.

3. P. Halmos, A Hilbert space problem book, Van Nostrand, Princeton, N.J., 1967. MR 34 \#8178.

4. M. Heins, Complex function theory, Pure and Appl. Math., vol. 28, Academic Press, New York, 1968. MR 39 \#413.

5. D. C. Kleinecke, On operator commutators, Proc. Amer. Math. Soc. 8 (1957), 535536. MR 19, 435.

6. C. Pearcy, Some unsolved problems on operator theory, Studies in Operator Theory, Math. Assoc. Amer. (to appear).

7. F. Riesz and B. Sz.-Nagy, Leçons d'analyse fonctionnelle, 2nd ed., Akad. Kiadó, Budapest, 1953; English transl., Ungar, New York, 1955. MR 15, 132; 17, 175.

8. F. V. Sirokov, Proof of a conjecture of Kaplansky, Uspehi Mat. Nauk 11 (1956), no. 4 (70), 167-168. (Russian) MR 19, 435.

9. J. G. Stampfli, Derications on $B(H)$ : The range (to appear).

10. J. P. Williams, On the range of a derivation, Pacific J. Math. 38 (1971), 273-279.

Department of Mathematics, Bucknell University, Lewisburg, Pennsylvania 17837 